\title{
CD10 expression in gastric adenocarcinoma and its correlation with histopathological features and lymph node metastasis
}

\author{
Bhavya Pappalamukalel Mohan', Sharath Kesavan Krishnan² \\ ${ }^{1}$ Associate Professor, Department of Pathology, Government Medical College, Thrissur, Kerala, India, ${ }^{2}$ Associate \\ Professor, Department of General Surgery, Government Medical College, Thrissur, Kerala, India
}

Background: Gastric cancer is one of the most common cancers worldwide. Invasion and metastasis are known prognostic factors. Previous studies have suggested CD10 expression in the epithelium and stroma of various carcinomas is associated with more aggressive behavior of the tumor. Aims and Objective: To study the immunohistochemical expression of CD10 in stromal cells of gastric adenocarcinoma and to correlate the expression with various clinicopathological features and lymph node metastasis. Materials and Methods: A cross sectional study of CD10 expression in 40 cases of gastric carcinoma in gastrectomy specimens was done. CD10 expression was correlated with age, gender, tumor site, tumor grade, histologic sub type, depth of invasion and lymph node status. Results: Out of 40 cases, $72.5 \%$ were males and $27.5 \%$ were females. Majority of cases were seen in $6^{\text {th }}$ and $7^{\text {th }}$ decades. Antrum was the most common location (70\%) and intestinal morphology was the commonest histologic subtype (47.5\%). Tumor size ranged from 2.5 to $11 \mathrm{cms}$. $40 \%$ tumors were well differentiated. Majority $(62.5 \%)$ of the tumors were in T3 stage. $25(62.5 \%)$ cases showed a positive CD10 expression in stromal cells. Stromal CD10 expression in gastric carcinoma did not correlate with the age and gender of the cases as well as the location and size of the tumor, histologic subtype and lymph node involvement but correlated with depth of invasion ( $T$ stage) $(p<0.05)$. Conclusion: CD10 expression in gastric adenocarcinoma shows a significant correlation with depth of invasion. CD10 may be used as an immunohistochemical surrogate of tumor behavior.

Key words: Gastric cancer; CD10; Invasion; Differentiation; Stroma;

Immunohistochemistry

\section{INTRODUCTION}

Gastric carcinoma is a leading cause of cancer globally which is responsible for over one million new cases in 2020 and an estimated 769,000 deaths (equating to one in every 13 deaths globally), ranking fifth for incidence and fourth for mortality worldwide. ${ }^{1}$ Men are twice commonly affected than women. ${ }^{1}$

India has a low incidence of gastric cancer and it is the $3^{\text {rd }}$ most common cancer in males and $6^{\text {th }}$ most common cancer in females. ${ }^{2}$ it is the second most common cause of cancer related deaths among Indian men and women in the age between 15 and 44 .
Etiology of gastric cancer includes Helicobacter pylori infection, diet and lifestyle, tobacco, alcohol and genetic susceptibility. The incidence of gastric cancer in India is low compared to developed countries, though there are certain geographical areas (Southern part and north eastern states of country) where the incidence is comparable to high-incidence areas of world. Despite the large number of patients being treated for gastric cancer, there are not sufficient publications discussing associated risk factors and outcomes in these patients. ${ }^{3}$

CD10 glycoprotein is a $100-\mathrm{kD}$ metalloendopeptidase located on cell surface. It inactivates a variety of 
biologically active extracellular matrix peptides involved in varied biological processes like tissue remodelling, embryogenesis, angiogenesis etc. CD10 is expressed by a variety of normal cell types, including lymphoid precursor cells, germinal center B lymphocytes and epithelial cells of lung, intestine, kidney, breast, placenta and many others. It was initially identified as the common acute lymphoblastic leukemia antigen (CALLA) followed by detection of this determinant on early precursors of B-cell line in bone marrow. Besides lymphoblastic leukemia and B-cell Non-Hodgkin lymphoma, CD10 expression has also been observed in cancers of head and neck, lung, breast, prostate, testis, liver and colorectum. ${ }^{4}$ Studies have showed correlation between level of CD10 expression and higher histological grade, larger tumor size, vascular invasion and overall survival rate in patients with solid tumors. ${ }^{5}$

Squamous cell carcinoma of head and neck, follicular and papillary thyroid cancer, melanomas, and esophageal carcinoma are reported to show a high CD 10 expression, whereas cervical carcinoma, and adenocarcinoma of the gastrointestinal tract have shown a low expression of CD10. These data highlights the use of CD10 as a significant predictive factor for tumor aggressiveness, therapy response and overall survival. ${ }^{6}$

Tumors induce a stromal reaction or desmoplasia in the course of its progression. Deregulated CD10 expression on epithelial cells as well as on surrounding stromal cells of tumor may lead to cell microenvironment disruption and tumor progression.

The present study aims to evaluate the immunohistochemical expression of CD10 in stromal cells of primary gastric adenocarcinoma (GAC) and to correlate the expression with age, gender, histopathological features (tumor site, tumor grade, histologic sub type, depth of invasion) and lymph node metastasis.

\section{MATERIALS AND METHODS}

This cross sectional study was performed at the department of Pathology, Government Medical College, Thrissur, Kerala, India. 40 cases of primary gastric adenocarcinoma diagnosed in gastrectomy specimens received in Pathology department during the study period (2018-2019) were included.

\section{Inclusion criteria}

1. All cases of primary gastric adenocarcinoma in gastrectomy specimens

2. All clinicopathological data (Age, gender, tumor site, tumor grade, histologic subtype, depth of invasion, lymph node status)

3. No neoadjuvant chemotherapy

\section{Exclusion criteria}

1. Gastrectomy performed after neoadjuvant chemotherapy

2. Non-carcinomatous gastric tumors

3. Gastric biopsies with a diagnosis of adenocarcinoma

4. Secondary tumors

\section{Methods}

The gastrectomy specimens were fixed in $10 \%$ formalin. Appropriate sections were obtained from tumor, adjacent mucosa, the site of maximum tumor invasion, resected margins and lymph nodes. Sections were then processed, paraffin embedded and stained with hematoxylin and eosin (H\&E). Tumor site (fundus, body, greater curvature, lesser curvature, antrum, pylorus), tumor grade (well differentiated, moderately differentiated, poorly differentiated grades), histologic type (intestinal, diffuse, others), maximum depth of invasion (T Stage) and lymph node metastasis (N Stage) were evaluated on all the specimens. A representative slide with advancing front of the tumor tissue stained with hematoxylin and eosin and the corresponding paraffin block were selected in each case.

\section{Immunohistochemistry}

In all 40 cases, 3 to 4 microns fresh sections obtained from the paraffin block with advancing front of the tumor tissue were taken on poly-L-lysine coated slides. These sections were subjected to immunohistochemistry (IHC) using mouse monoclonal antiCD10 antibody (Clone GM003) by following the manufacturer's protocol. Tris-EDTA Buffer was used for antigen retrieval by multi epitope heat retrieval method. Incubation with CD10 primary antibody was done for 60 minutes. Poly Excel Horseradish peroxidase (HRP) / Diaminobenzidine (DAB) detection system was used for staining. Lymph node section was used as positive control. Additional tumor section stained without primary antibody was used as negative control.

\section{Evaluation of immunostaining}

Percentages staining of stromal cells were evaluated with $10 \%$ as cut off. Stromal cells with more than $10 \%$ membrane positivity were considered positive and those with less than $10 \%$ were taken as negative.

\section{Statistical analysis}

Correlation between CD10 expression of stromal cells and histopathological factors was evaluated using the chi square test or Fischer's exact test. P-values $<0.05$ were considered significant.

\section{Ethics}

The study was approved by Institutional Ethics Committee (B6-8772/2016/MCTCR). 


\section{RESULTS}

Out of the 40 cases, 29 cases were males (72.5\%) and 11 were females $(27.5 \%)$. The male to female ratio is 2.6:1 (Figure 1).

The age of the patients ranged from 39 years to 81 years. Majority of the patients were in sixth and seventh decades of life followed by $>70$ years and $<50$ years of age (Figure 2 ).

Tewnty-eight tumors were located in the antrum (70\%), 10 in the pylorus $(25 \%)$ and 1 each in the cardia and greater curvature $(2.5 \%)$. Half of the cases $(n=20 ; 50 \%)$ had a tumor size of $<5 \mathrm{cms}$ and the rest of the cases $(n=20$; $50 \%$ ), it was $>5 \mathrm{cms}$. (Range of tumor size: 2.5 to $11 \mathrm{cms}$ ) (Tables 1 and 2).

Among the 40 tumors, 16 (40\%) were well differentiated grade, 12 cases each $(30 \%)$ were moderately differentiated and poorly differentiated grades respectively (Table 3 ).

Histologically, 19 (47.5\%) tumors had intestinal morphology, $16(40 \%)$ showed diffuse morphology and 3 of them were mucinous adenocarcinomas $(7.5 \%)$. One tumor $(2.5 \%)$ showed papillary morphology, another one $(2.5 \%)$ was an early gastric carcinoma.

The depth of invasion (T Stage) was defined as: ${ }^{7}$ [T1 =Tumor invades the lamina propria, muscularis mucosae or submucosa

\section{Table 1: Distribution of cases according to site}

\begin{tabular}{lcc}
\hline Tumor site & Frequency & Percent \\
\hline Antrum & 28 & 70 \\
Cardia & 1 & 2.5 \\
Greater curvature & 1 & 2.5 \\
Pylorus & 10 & 25 \\
Total & 40 & 100 \\
\hline
\end{tabular}

Table 2: Distribution of cases as per tumor size

\begin{tabular}{lcc} 
Tumor size $\mathbf{( c m )}$ & Frequency & Percent \\
\hline$\leq 5.0$ & 20 & 50 \\
$>5$ & 20 & 50 \\
Total & 40 & 100 \\
\hline
\end{tabular}

\begin{tabular}{|c|c|c|}
\hline Differentiation/Grade & Frequency & Percent \\
\hline WD & 16 & 40 \\
\hline MD & 12 & 30 \\
\hline PD & 12 & 30 \\
\hline Total & 40 & 100 \\
\hline
\end{tabular}

T2 $=$ Tumor invades the muscularis propria

T3 =Tumor penetrates the sub serosal connective tissue without invasion of the visceral peritoneum or adjacent structures

T4 =Tumor invades the serosa or adjacent structures]

In our study, 2 cases (5\%) were in T1 stage, 5 tumors $(12.5 \%)$ in T2 stage, 25 tumors $(62.5 \%)$ in T3 stage and 8 cases $(20 \%)$ were in T4 stage.

There were $32(80 \%)$ cases showing lymph node metastases and $8(20 \%)$ cases without lymph node metastases (N0 stage). Out of these 32 tumors with lymph node metastasis, $15(37.5 \%)$ were in N1, $9(22.5 \%)$ in N2 and $8(20 \%)$ were in N3 stages.

\section{Stromal CD10 Expression}

Out of 40 tumors studied, 25 (62.5\%) showed a positive CD10 expression in stromal cells (Figure 3). Remaining $15(37.5 \%)$ cases were negative (Table 4$)$. No immunostaining was seen in the stromal cells of adjacent normal tissue. We noted a parallel staining in the tumor epithelial cells in some cases, but it was cytoplasmic (not membranous).

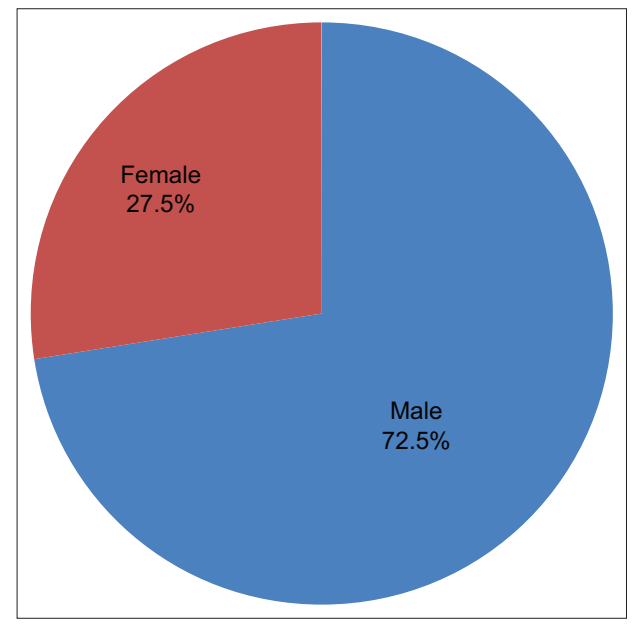

Figure 1: Gender distribution of cases

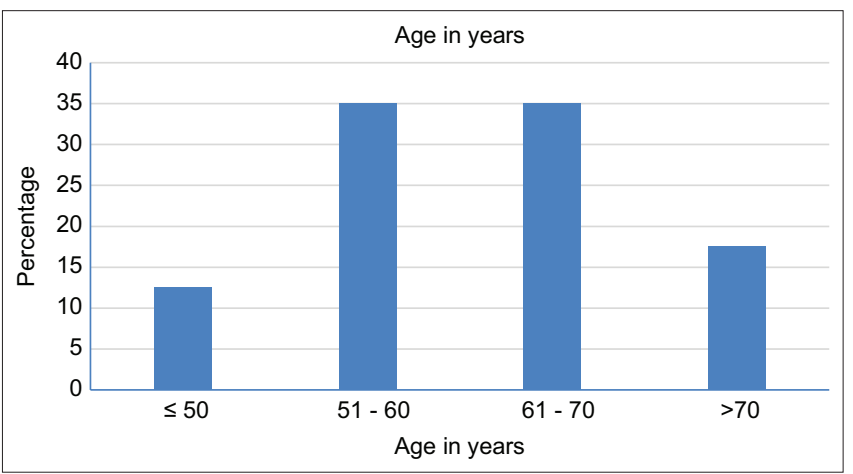

Figure 2: Age distribution of cases 


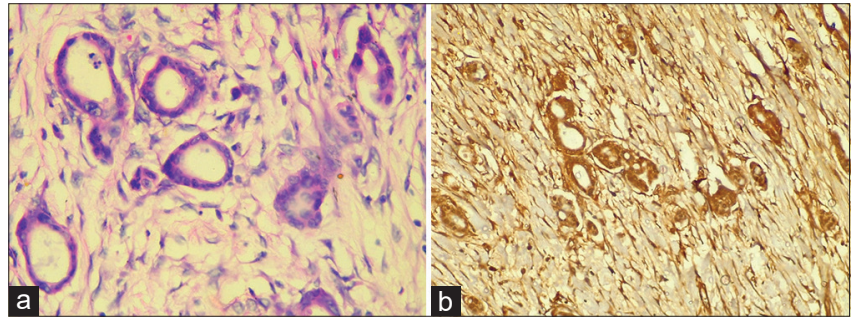

Figure 3: (a) Gastric adenocarcinoma, intestinal type (H\&Ex400). (b) CD10 positivity in stromal cells (IHCx200)

\begin{tabular}{lcc}
$\begin{array}{l}\text { Table 4: CD10 expression in Gastric } \\
\text { adenocarcinoma }\end{array}$ & \\
\hline Stromal CD10 & Frequency & Percent \\
\hline Positive & 25 & 62.5 \\
Negative & 15 & 37.5 \\
Total & 40 & 100 \\
\hline
\end{tabular}

\section{10 versus Tumor Site}

Of the 28 tumors at antrum, CD10 expression was noted in 17 cases $(68 \%)$ and 11 cases $(73.3 \%)$ were CD10 negative. One tumor (4\%) each at cardia and greater curvature was CD10 positive. Among the 10 pylorus tumors, 6 (24\%) were CD 10 positive and 4 (26.7\%) were CD10 negative.

\section{10 versus Tumor Grade}

There were 16 well differentiated tumors, of which $8(32 \%)$ were CD 10 positive and $8(53.3 \%)$ were CD10 negative. In the 12 moderately differentiated tumors, $9(36 \%)$ showed CD 10 positivity and 3 (20\%) were CD10 negative. Out of the 12 poorly differentiated tumors, $8(32 \%)$ were CD10 positive and $4(26.7 \%)$ were CD10 negative.

\section{10 versus T Stage (depth of invasion)}

Out of 40 tumors, $33(82.5 \%)$ tumors belonged to a high tumor stage of T3 and T4. Among these, 23 (92\%) were CD10 positive and $9(66.7 \%)$ were CD10 negative. In the remaining $7(17.5 \%)$ tumors which were in T1 and T2 stages, $2(8 \%)$ showed CD10 expression and $5(33.3 \%)$ tumors did not.

\section{10 versus $\mathrm{N}$ stage}

There were $32(80 \%)$ cases showing lymph node metastases and $8(20 \%)$ cases without lymph node metastases. Among the 32 node positive cases, 22 (88\%) were CD10 positive and $10(66.7 \%)$ were CD10 negative. Of the $8 \mathrm{~N} 0$ stage cases, $3(12 \%)$ cases showed CD10 expression and $5(33.3 \%)$ tumors were negative.

A p-value of 0.041 was noted with a significant correlation between stromal CD10 expression and T stage (depth of invasion) of the tumor. There was no statistically significant correlation between CD10 expression and age, gender, tumor size and site, histologic grade, histologic subtype and $\mathrm{N}$ stage (Table 5).

\section{DISCUSSION}

The global cancer burden is expected to be 28.4 million cases in 2040, a 47\% rise from 2020. ${ }^{1}$ Gastric carcinoma remains an important contributor to this burden. Recent notable findings are the increase in the incidence of stomach cancer (cardia and noncardia gastric cancers combined) among young adults (aged $<50$ years) in both low risk and high risk countries. ${ }^{1}$ Many of the gastric cancers present at an advanced stage, with lymph node metastasis at diagnosis, which generally contributes to the poor outcome. Recent studies on cancer research is focusing on the newer prognostic markers and targeted therapies.

CD10 antigen is a surface zinc metalloendopeptidase and is expressed in various normal and tumor cells of lymphoid and non-lymphoid origin. Studies have shown that CD10 expression in intratumoral stromal cells may contribute to tumor progression in various cancers. When cancer cells invade and metastasize, the number of stromal cells increases. The CD10 positive stromal cells are present at the invasive front of the tumor suggests the interaction between cancer cells and CD10-positive stromal cells. Most studies measured epithelial CD10 expression by immunohistochemistry and cDNA array, but few studies measured stromal CD10 expression by immunohistochemistry.

Kadota $\mathrm{K}$ et al. reviewed tumor slides of resected pathologic stage I lung adeno-carcinomas and analyzed CD10 expression by IHC using tissue microarrays. They stated that in stage I lung adenocarcinoma, tumoral CD10 correlated with high-grade histology and was an independent predictor of recurrence in intermediate-grade tumors. ${ }^{8}$

Bilalovic N. et al. concluded that CD10 expression in malignant melanoma is associated with tumor progression. They evaluated CD10 expression by IHC in tumor and stromal cells in 70 biopsies with primary and 28 with metastatic malignant melanomas. ${ }^{9}$

Louhichi T et al., studied CD10 expression by IHC in a series of 133 invasive breast carcinoma cases and they supported the role of stromal CD10 expression in breast cancer progression and dissemination, and suggest a relationship with cancer stem cells. ${ }^{10}$

Utility of CD10 as a significant predictive factor is suggested in different cancers like squamous cell carcinoma 


\begin{tabular}{|c|c|c|c|c|c|c|c|c|c|}
\hline \multirow[t]{3}{*}{ No } & \multirow[t]{3}{*}{ Variable } & \multirow[t]{3}{*}{ Types } & \multirow[t]{3}{*}{ Total number } & \multirow[t]{3}{*}{$\%$} & \multicolumn{4}{|c|}{ CD10 } & \multirow[t]{3}{*}{ p Value } \\
\hline & & & & & \multicolumn{2}{|c|}{ Positive } & \multicolumn{2}{|c|}{ Negative } & \\
\hline & & & & & $\mathbf{N}$ & $\%$ & $\mathbf{N}$ & $\%$ & \\
\hline \multirow[t]{4}{*}{1} & Tumor site & antrum & 28 & 70 & 17 & 68 & 11 & 73.3 & 0.738 \\
\hline & & cardia & 1 & 2.5 & 1 & 4 & 0 & 0.0 & \\
\hline & & Greater curvature & 1 & 2.5 & 1 & 4 & 0 & 0.0 & \\
\hline & & pylorus & 10 & 25 & 6 & 24 & 4 & 26.7 & \\
\hline \multirow[t]{3}{*}{2} & Tumor grade & WD & 16 & 40 & 8 & 32 & 8 & 53.3 & 0.376 \\
\hline & & MD & 12 & 30 & 9 & 36 & 3 & 20.0 & \\
\hline & & PD & 12 & 30 & 8 & 32 & 4 & 26.7 & \\
\hline \multirow[t]{2}{*}{3} & T Stage (depth of invasion) & $\mathrm{T} 1$ and $\mathrm{T} 2$ & 7 & 17.5 & 2 & 8.0 & 5 & 33.3 & 0.041 \\
\hline & & T3 and T4 & 33 & 82.5 & 23 & 92.0 & 10 & 66.7 & \\
\hline \multirow[t]{2}{*}{4} & Lymph node metastasis & Absent & 8 & 20 & 3 & 12 & 5 & 33.3 & 0.126 \\
\hline & & Present & 32 & 80 & 22 & 88 & 10 & 66.7 & \\
\hline
\end{tabular}

of head and neck, follicular papillary and papillary thyroid cancer, esophageal carcinoma, colonic carcinoma and cervical carcinoma. ${ }^{6}$ However, there are not much studies on $\mathrm{CD} 10$ expression in GAC.

In our study, stromal CD10 expression was analyzed by IHC on formalin fixed paraffin embedded sections. We noted a statistically significant correlation between depth of invasion ( $T$ stage) in GAC and CD10 expression. However, age, gender, tumor site, tumor grade and lymph node metastasis were not statistically related to CD10 expression in our study. In a similar study by Forooshani MK et al. a statistically significant correlation was detected between tumor stage and CD10 expression. ${ }^{11}$ This suggests that CD10 expression by stromal cells may play an important role in the pathogenesis of gastric cancer and also that the proliferation of CD10-positive stromal cells is part of the mechanism of invasion in gastric cancer.

Chinthakindi S.et al demonstrated a significant correlation between stromal CD10 expression and lymph nodal metastasis and no significant correlation with differentiation, invasion and TNM stage. ${ }^{12}$

Huang WB et al. have shown that stromal cells expressing CD10 may play an important role in gastric carcinogenesis. CD10 expression by stromal cells seems to promote invasion and metastasis of differentiated gastric carcinoma. ${ }^{13}$

Carl MC Grath et al., provided strong evidence that CD10 is upregulated in GAC and lymph node metastases and that the ability of CD10 to degrade gastrointestinal peptides may play an important role in pathogenesis of gastric carcinoma. ${ }^{14}$

Our study showed a significant correlation between stromal CD10 expression and depth of invasion in GAC, which becomes important in the mechanism of tumor invasion and metastasis, predicting a poor biological behavior.
Future large cohort studies on the molecular basis of CD10 expression in stromal cancer interaction in GAC will be helpful to develop new therapeutic strategies.

\section{CONCLUSION}

Depth of invasion ( $\mathrm{T}$ stage) in gastric adenocarcinoma is having a significant correlation with stromal CD10 expression. CD10 may be considered as a useful IHC surrogate to predict outcomes in GAC.

\section{ACKNOWLEDGEMENT}

The authors would like to acknowledge patients and their families, Dr. Feroze M, Retired HOD and Dr. Prasad P.H, Present HOD, Pathology Department, for their support and motivation, Dr. Ravindran Chirukandath, Chairman, IEC, for the administrative support, Dr. Gokulapriya, Senior resident, for her help in image acquisition and the staff of histopathology laboratory of our institution.

\section{REFERENCES}

1. Sung H, Ferlay J, Siegel RL, Laversanne M, Soerjomataram I, Jemal A, et al. Global cancer statistics 2020: GLOBOCAN estimates of incidence and mortality worldwide for 36 cancers in 185 countries. CA Cancer J Clin. 2021; 71(3):209-249. https://doi.org/10.3322/caac.21660

2. Servarayan Murugesan $C$, Manickavasagam $K$, Chandramohan A, Jebaraj A, Jameel ARA, Jain MS, et al. Gastric cancer in India: epidemiology and standard of treatment. Updates in Surgery. 2018; 70 (2):233-239. https://doi.org/10.1007/s13304-018-0527-3

3. Dikshit RP, Mathur G, Mhatre $S$ and Yeole BB. Epidemiological review of gastric cancer in India. Indian J Med Paediatr Oncol. 2011; 32 (1):3-11.

https://doi.org/10.4103/0971-5851.81883

4. Mishra D, Singh S and Narayan G. Role of B Cell Development Marker CD10 in Cancer Progression and Prognosis. Molecular 
Biology International.2016; 4328697.

https://doi.org/10.1155/2016/4328697

5. S. Thomas, R. J. Babu, K. Agarwal, V. Puri, M. Jain, M. Andley, et al. Effect of neoadjuvant chemotherapy on stromal CD10 antigens in breast cancer - a preliminary study. Indian $\mathrm{J}$ Cancer. 2013; 50(1): 46-51.

https://doi.org/10.4103/0019-509X.112299

6. Wlodek J. Clinical significance of CD10 expression in cancer. Int Clin Pathol J. 2017; 5(1):192-194.

https://doi.org/10.15406/icpjl.2017.05.00121

7. Amin MB, Edge S, Greene F, Byrd DR, Brookland RK, Washington MK, et al. (Eds.) AJCC Cancer Staging Manual (8 ${ }^{\text {th }}$ edition). Springer International Publishing: American Joint Commission on Cancer. 2017; 211pp.

8. Kadota K, Buitrago D, Lee MC, Vargas JV, Sima CS, Jones DR, et al. Tumoral CD10 expression correlates with high-grade histology and increases risk of recurrence in patients with stage I lung adenocarcinoma. Lung Cancer. 2015; 89(3):329-336. https://doi.org/10.1016/j.lungcan.2015.06.003

9. Bilalovic N, Sandstad B, Golouh R, Nesland JM, Selak and Torlakovic EE. CD10 protein expression in tumor and stromal cells of malignant melanoma is associated with tumor progression. Mod Pathol. 2004; 17(10):1251-1258. https://doi.org/10.1038/modpathol.3800174

10. Louhichi T, Saad H, Dhiab MB, Ziadi S and Trimeche M. Stromal CD10 expression in breast cancer correlates with tumor invasion and cancer stem cell phenotype. BMC Cancer. 2018; 18(1):49. https://doi.org/10.1186/s12885-017-3951-8

11. Forooshani MK, Jafarian $A H$, Takallou $L$ and Mohamadian Roshan, N. CD10 expression in gastric carcinoma is correlated with tumor grade and survival. Univ Med 2019; 38:41-47. https://doi.org/10.18051/UnivMed.2019.v38.41-47

12. Sravan C, Kanna S, Kumar MK. Expression of CD10 Marker in Stromal Cells of Gastric Carcinoma: A Prospective Study Sch. Acad. J. Biosci. 2017; 5(3):192-199.

https://doi.org/10.21276/sajb.2017.5.3.11

13. Huang WB, Zhou XJ, Chen JY, Zhang LH, Meng K, Ma HH, et al. CD10-positive Stromal Cells in Gastric Carcinoma: Correlation with Invasion and Metastasis. Jpn J Clin Oncol. 2005; 35 (5) 245- 250. https://doi.org/10.1093/jjco/hyi076

14. Carl-McGrath S, Lendeckel U, Ebert M, Wolter AB, Roessner A and Rocken C. The ectopeptidases CD10, CD13, CD26, and CD143 are upregulated in gastric cancer. Int J Oncol. 2004; 25: $1223-1232$.

https://doi.org/10.3892/ijo.25.5.1223

\section{Author's Contribution:}

BPM- Concept and design of the study, data collection, literature search and review, manuscript preparation and result interpretation; SKK- Concept and coordination of the study, review of literature, helped in preparing first draft of manuscript and critical revision of the manuscript.

\section{Work Attributed to}

Government Medical College, Thrissur, Kerala, India.

Orcid ID:

Dr. Bhavya Pappalamukalel Mohan- (1D https://orcid.org/0000-0002-0262-0778

Dr. Sharath Kesavan Krishnan- (iD https://orcid.org/0000-0003-0477-9659 